STRUCTURAL

BIOLOGY

ISSN 2059-7983

\title{
Diffraction structural biology - an introductory overview
}

\author{
Atsushi Nakagawa, ${ }^{a *}$ John R. Helliwell ${ }^{\mathrm{b} *}$ and Yuriko Yamagata ${ }^{\mathrm{c} *}$ \\ a Institute for Protein Research, Osaka University, 3-2 Yamadaoka, Suita, Osaka 565-0871, Japan, ${ }^{\mathbf{b}}$ Department of \\ Chemistry, The University of Manchester, Brunswick Street, Manchester M13 9PL, United Kingdom, and ${ }^{\mathbf{c}}$ Kumamoto \\ University, 5-1 Oe-honmachi, Chuo-ku, Kumamoto 862-0973, Japan. *Correspondence e-mail: \\ atsushi@protein.osaka-u.ac.jp, john.helliwell@manchester.ac.uk, yamagata@gpo.kumamoto-u.ac.jp
}

Keywords: diffraction structural biology; ISDSB2019.

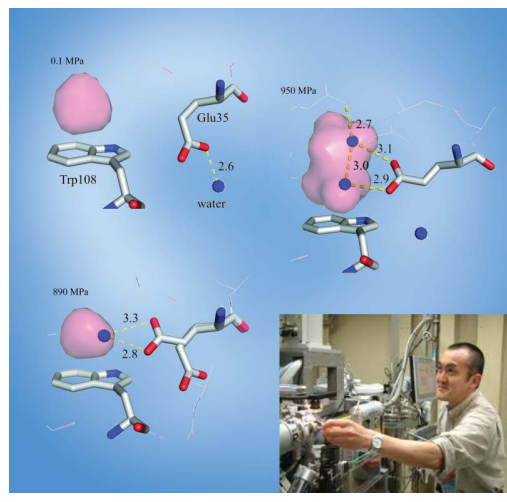

The virtual special issue now available at https://journals.iucr.org/special_issues/2021/ ISDSB2019 collects together articles published in Acta Crystallographica Section D and Acta Crystallographica Section F submitted in the context of the 6th International Symposium on Diffraction Structural Biology (ISDSB) 2019, which was held at the Osaka University Hall in Osaka, Japan on 17-19 October 2019. The ISDSB series of symposia was initiated in 2003 by the Japan Society for the Promotion of Science (JSPS) and specifically by the University-Industry Cooperative Research Committee (\#169) (169th Committee) chaired by Professor Noriyoshi Sakabe. The phrase 'diffraction structural biology' was proposed by the 169th Committee and has shown to the world the importance of research on structural biology mainly by X-ray, neutron, electron and other diffraction techniques. In 2003 the 1st International Symposium on Diffraction Structural Biology (ISDSB2003) was held in Tsukuba hosted by the 169th Committee, followed by the 2nd international symposium (ISDSB2007) in Tokyo in 2007, ISDSB2010 in Paris and ISDSB2013 in Nagoya. The previous conference was held in Knoxville, Tennessee, USA, close to the Oak Ridge National Laboratory (ORNL) neutron user facilities, the High Flux Isotope Reactor (HFIR) and Spallation Neutron Source (SNS) in 2016. The special issue of Acta Crystallographica Section D, Volume 74, Part 8 (2018) presented the articles submitted in the context of the ISDSB2016.

The central theme of the series of ISDSB conferences is to 'bring structural biologists using diffraction, and the scientists using a wide range of other technologies, closer together as well as stimulate the interface of basic research and industrial applications'. Structural biology has clarified, even exposed, the mechanisms of action of molecular machines responsible for life phenomena through the three-dimensional structures of biological macromolecules. In order to recognize the specificity found in biological macromolecules, which are essential for life, related to disease and drug discovery, it is also necessary to embrace other techniques complementary to the diffraction method. Both domains have advanced enormously. In recent years, diffraction capabilities have advanced greatly - the X-ray free-electron laser facility SACLA and powerful pulse neutron facility J-PARC have been in operation in Japan, and similar facilities are being constructed all over the world. There are also massive advances in synchrotron X-ray sources in achieving extreme levels of brightness, Also, in the complementary domain of microscopy a big breakthrough has been achieved in electron microscopy in recent years. Indeed the Nobel Prize for Chemistry was awarded in 2017 to Jacques Dubochet, Joachim Frank and Richard Henderson for cryo-electron microscopy developments. The ISDSB2019 symposium played an important role based on this new era, and in addition to structural analysis based on diffraction phenomena as a core technology, other scattering, dynamics and computational science developments were presented at the Symposium. A total of 126 participants, with many young scientists and $\mathrm{PhD}$ students, from 12 countries took part. The speakers included one Nobel Lecture by Joachim Frank, three Plenary Lecturers (Ian Wilson, Yoshinori Fujiyoshi and Stephen K. Burley), 19 invited speakers, 13 selected oral speakers, 1 sponsored session speaker and 49 poster presentations. Scientific session topics included MX facilities, macromolecular crystallography, cryo-TEM (including single-particle analysis and microED), software and databases, and drug discovery research. High-school students also attended the Nobel Lecture session and gave poster presentations in the symposium, which were of a 
delightful high quality and with the presenters asking probing questions of the visitors to their posters. Three lunchtime seminars were held and sponsored by JEOL, Bruker and Thermo Fisher Scientific.

A very sad context to the meeting was the death of our close colleague Professor Nobuhisa Watanabe, who died from leukemia on 26 March 2019 at the age of 57. The opening session of ISDSB2019 was dedicated to him and we highlighted his scientific achievements in protein crystallography and beamline instrumentation. An image of him and his work has been chosen to illustrate this special issue. Marking Nobuhisa's memory stimulated us all to focus on the natural living organism and its preferred temperature and pressure.

The symposium was supported jointly by the 169th Committee of the JSPS and by many industrial companies as well as generous individuals. We also wish to express our sincere thanks to the University-Industry Cooperation and Research Program Division of the JSPS, to Osaka University, and to all companies and persons who contributed their donations and cooperation. 